05,11

\title{
Особенности состояний фторида кобальта в окрестности критического поля
}

\author{
(C) О.Г. Медведовская ${ }^{1}$, Т.А. Федоренко ${ }^{2}$, Г.К. Чепурных ${ }^{3, \uparrow}$ \\ ${ }^{1}$ Сумский государственный педагогический университет им. А.С. Макаренко, \\ Сумы, Украина \\ ${ }^{2}$ Сумский государственный университет, \\ Сумы, Украина \\ ${ }^{3}$ Институт прикладной фризики, \\ Сумы, Украина \\ ฯ E-mail: ipfmail@ipfcentr.sumy.ua
}

(Поступила в Редакцию 12 января 2016 г.

В окончательной редакции 28 апреля 2016 г.)

С учетом роста экспериментальных исследований, связанных с использованием фторида кобальта, изучено состояние фторида кобальта в окрестности критического значения $H_{c}$ продольного магнитного поля $\mathbf{H}$, при котором магнитная подсистема кристалла $\mathrm{CoF}_{2}$ с большим взаимодействием Дзялошинского переходит из антиферромагнитной фазы в угловую. Обнаружено, что, несмотря на необычайно большую величину магнитной анизотропии кристалла, состояние магнитной подсистемы при $H=H_{c}$ оказалось крайне чувствительным к незначительному отклонению вектора $\mathbf{H}$ от оси $C_{4}$. Другая особенность состоит в том, что эта высокая чувствительность исчезает при увеличении или уменьшении магнитного поля всего лишь на тысячные доли $H_{c}$. Выполненные исследования применимы к магнитоупорядоченным кристаллам $\mathrm{FeF}_{3}$, $\mathrm{Cu}_{2} \mathrm{OSeO}_{3}$, которые, так же как и $\mathrm{CoF}_{2}$, обладают сильным взаимодействием Дзялошинского и значительной магнитной анизотропией. Обнаруженная аномалия в уменьшении эффективной магнитной анизотропии представляет интерес в связи с многочисленными попытками уменьшить магнитную анизотропию в кристаллах с гигантской магнитострикцией, которые необходимы для использования в качестве сенсоров и вибраторов.

\section{1. Введение}

В последние годы увеличилось количество экспериментальных исследований [1-6], связанных с использованием фторида кобальта $\left(\mathrm{CoF}_{2}\right)$. Однако особенностью кристалла $\mathrm{CoF}_{2}[7,8]$, как и кристаллов $\mathrm{FeF}_{3}, \mathrm{Cu}_{2} \mathrm{OSeO}_{3}$, которым также уделяется внимание (см., например, [9,10] соответственно), является наличие необычайно большой магнитной анизотропии и сильного взаимодействия Дзялошинского. Это обстоятельство создавало (и создает) определенные трудности для экспериментального изучения указанных кристаллов, и поэтому возникает необходимость в более детальном теоретическом изучении данных кристаллов на примере $\mathrm{CoF}_{2}$.

Согласно экспериментальным данным [7,8], особенностью кристалла $\mathrm{CoF}_{2}$ (и, по-видимому, кристалла $\left.\mathrm{FeF}_{3}\right)$ является то, что под влиянием продольного магнитного поля магнитная подсистема этого кристалла вместо обычно наблюдаемого перехода из состояния $\mathbf{l}\|\mathbf{A}\|[001]$ в состояние $\mathbf{I} \perp \mathbf{A}(\mathbf{l}-$ вектор антиферромагнетизма, $\mathbf{A}$ - ось легчайшего намагничивания) переходит в угловую фазу. Кроме того, этот кристалл обладает значительной магнитострикцией [11]. Поскольку обнаруженный экспериментально $[7,8]$ переход в угловую фазу вызвал много вопросов, в работах $[12,13]$ были выполнены теоретические исследования. И в них было показано, что переход в угловую фазу являет- ся общим свойством всех легкоосных тетрагональных антиферромагнетиков (не зависимо от того, влияет магнитное поле на намагниченность подрешеток или нет), в которых в базисной плоскости преобладает анизотропия, обусловленная взаимодействием Дзялошинского, а не обменно-усиленная анизотропия четвертого порядка.

Одна из трудностей численного решения уравнений, определяющих состояние магнитной подсистемы только в продольном магнитном поле, состоит в значительном разбросе констант плотности энергии, определенных в различных экспериментах $[7,8]$.

Расчетных работ, определяющих состояние магнитной подсистемы при отклонении направления магнитного поля от оси $C_{4}$ в окрестности поля $H_{c}$ фазового перехода, не существует. Тогда как экспериментальные данные, полученные еще в работе [8], свидетельствуют об особенностях поведения магнитной подсистемы при отклонениях направления магнитного поля от оси $C_{4}$ всего на несколько угловых минут. Поэтому целью настоящей работы является выяснение возможных аномалий в уменьшении эффективной магнитной анизотропии в окрестности критического поля $H_{c}$. Это важно и по той причине, что в настоящее время существует огромный поток экспериментальных исследований, направленных на создание высокочувствительных сенсоров, генераторов мощного звука и ультразвука, нажимных устройств путем использования материалов, 
обладающих гигантской магнитострикцией (см., например, [14-16] соответственно).

Этот интерес возник сразу после обнаружения [17-19] при низких температурах в редкоземельных металлах тербия и диспрозия анизотропной магнитострикции $\Delta l / l \sim 10^{-2}$. И хотя гигантская магнитострикция затем была обнаружена в интерметаллических соединениях $\mathrm{TbFe}_{2}$ и $\mathrm{DyFe}_{2}[20]$ при температурах выше комнатной, из-за огромной магнитной анизотропии гигантская магнитострикция реализовывалась при очень сильных изменениях магнитного поля. Попытки синтезировать материалы с малой магнитной анизотропией приводили к уменьшению магнитострикции (более подробно см. обзор [21]).

\section{2. Составление уравнений}

Поскольку рассматривается область магнитных полей, близких к критическому значению $H_{c}$, плотность энергии $\mathscr{H}$, используя данные работы [8], запишем в форме

$$
\begin{aligned}
\mathscr{H}= & 2 M_{0}\left[\frac{1}{2} E \mathbf{m}^{2}+\frac{1}{2} G(\mathbf{m l})^{2}\right. \\
& \left.+D\left(m_{x} l_{y}+l_{x} m_{y}\right)+\frac{1}{2} A_{1}\left(l_{x}^{2}+l_{y}^{2}\right)-\mathbf{m H}\right],
\end{aligned}
$$

где $\mathbf{m}=\left(\mathbf{M}_{1}+\mathbf{M}_{2}\right) / 2 M_{0}, \mathbf{l}=\left(\mathbf{M}_{1}-\mathbf{M}_{2}\right) / 2 M_{0}-$ феррои антиферромагнитные векторы, $\mathbf{M}_{1}$ и $\mathbf{M}_{2}-$ намагниченности подрешеток, $2 M_{0}$ - намагниченность насыщения, $E$ и $G-$ константы обменного взаимодействия, $D$ - константа взаимодействия Дзялошинского, $A_{1}-$ константа одноосной анизотропии, $\mathbf{H}-$ внешнее магнитное поле.

Согласно выбранной форме записи, все константы являются эффективными полями и измеряются в эрстедах. В выражении (1) учтены только главные члены, влияющие на состояние магнитной подсистемы в окрестности $H_{c}$. На векторы $\mathbf{m}$ и $\mathbf{I}$ наложено условие связи

$$
\mathbf{m}^{2}+\mathbf{l}^{2}=1,
$$

а условие связи $\mathbf{m} \cdot \mathbf{l}=0$ невыполнимо из-за зависимости намагниченности подрешеток $\mathbf{M}_{1}$ и $\mathbf{M}_{2}$ от магнитного поля.

Ограничимся полями, при которых возможно выполнение условия $m^{2} \ll 1$. На основе уравнения

$$
\partial \mathscr{H} / \partial \mathbf{m}=0
$$

в работе [8] определено выражение для намагниченности $\mathbf{m}$. В этой же работе [8] из плотности энергии (1) исключено $\mathbf{m}$ и получено выражение для плотности энергии в плоскости $Z X$. Для нашего случая указанная формула в переменных $\theta$ и $\varphi(\theta$ и $\varphi-$ полярный и азимутальный углы вектора I) имеет вид

$$
\begin{aligned}
\mathscr{H}= & 2 M_{0}\left\{-\frac{1}{2 E}\left[H_{x}^{2}+H_{z}^{2}-2 H_{x} D \sin \theta \sin \varphi\right.\right. \\
& \left.+D^{2} \sin ^{2} \theta\right]+\frac{G}{2 E(E+G)}\left[H_{x}^{2} \sin ^{2} \theta \cos ^{2} \varphi\right. \\
& +H_{x} H_{z} \sin 2 \theta \cos \varphi+H_{z}^{2} \cos ^{2} \theta-2 H_{x} D \\
& \times \sin ^{3} \theta \sin 2 \varphi \cos \varphi-2 H_{z} D \sin ^{2} \theta \cos \theta \sin 2 \varphi \\
& \left.\left.+D^{2} \sin ^{4} \theta \sin ^{2} 2 \varphi\right]+\frac{1}{2} A_{1} \sin ^{2} \theta\right\} .
\end{aligned}
$$

Используя (4), получаем уравнения относительно $\theta$ и $\varphi$

$$
\begin{aligned}
\frac{\partial \mathscr{H}}{\partial \varphi}= & \frac{1}{E} H_{x} D \sin \theta \cos \varphi+\frac{G}{2 E(E+G)} \\
& \times\left[-H_{x}^{2} \sin ^{2} \theta \sin 2 \varphi-H_{x} H_{z} \sin 2 \theta \sin \varphi\right. \\
& -4 H_{x} D \sin ^{3} \theta \cos 2 \varphi \cos \varphi+4 H_{x} D \\
& \times \sin ^{3} \theta \sin ^{2} \varphi \cos \varphi-4 H_{z} D \sin ^{2} \theta \cos \theta \\
& \left.\times \cos 2 \varphi+4 D^{2} \sin ^{4} \theta \sin 2 \varphi \cos 2 \varphi\right], \\
\frac{\partial \mathscr{H}}{\partial \theta}=\frac{1}{E} & H_{x} D \cos \theta \sin \varphi-\frac{1}{2 E} D^{2} \sin 2 \theta \\
+ & \frac{G}{2 E(E+G)}\left[H_{x}^{2} \sin 2 \theta \cos { }^{2} \varphi+2 H_{x} H_{z} \cos 2 \theta \cos \varphi\right. \\
- & H_{z}^{2} \sin 2 \theta-3 H_{x} D \sin 2 \theta \sin \theta \sin 2 \varphi \cos \varphi \\
- & 2 H_{z} D \sin 2 \theta \cos \theta \sin 2 \varphi+2 H_{z} D \sin ^{3} \theta \cos \theta \sin 2 \varphi \\
+ & \left.2 D^{2} \sin 2 \theta \sin ^{2} \theta \sin 2 \varphi\right]+\frac{1}{2} A_{1} \sin ^{2} 2 \theta
\end{aligned}
$$

\section{3. Решение уравнений}

В случае $\mathbf{H} \| \mathbf{C}_{4}$ уравнения относительно углов $\theta$ и $\varphi$ для любых легкоосных тетрагональных антиферромагнетиков в угловой фазе имеют решение $\varphi=\pi / 4$, которое соответствует минимуму энергии $[12,13]$. Если составляющая $H_{x} \neq 0$, то можно положить

$$
\varphi=\pi / 4+\varphi_{0}
$$

и при $H_{x} \ll H_{z}$ считать $\varphi_{0} \ll 1$.

В этом случае, производя разложения тригонометрических функций в ряд и удерживая необходимые слагаемые, для угла $\theta$ получаем уравнение

$$
\begin{aligned}
\frac{1}{\sqrt{2}} H_{x} & {\left[\left(1+\frac{E}{G}\right) D \cos \theta+H_{z} \cos 2 \theta\right] } \\
+ & \sin \theta\left\{\operatorname { c o s } \theta \left[-H_{z}^{2}-2 H_{z} D \cos \theta+2 D^{2} \sin ^{2} \theta\right.\right. \\
& \left.\left.+\left(1+\frac{E}{G}\right)\left(A_{1} E-D^{2}\right)\right]+H_{z} D \sin ^{2} \theta\right\}=0 .
\end{aligned}
$$


Если $H_{x}=0$ (продольное магнитное поле), то из (8) следует

$$
\begin{aligned}
\cos \theta[ & -H^{2}-2 H D \cos \theta+2 D^{2} \sin ^{2} \theta \\
& \left.+\left(1+\frac{E}{G}\right)\left(A_{1} E-D^{2}\right)\right]+H D \sin ^{2} \theta=0 .
\end{aligned}
$$

Уравнение (9) совпадает с уравнением (10) из работы [8]. Но это уравнение содержит четыре константы $\left(E, G, A_{1}\right.$ и $\left.D\right)$, и их значения, определенные в различных экспериментах, оказались разбросанными. Согласно $[7,8]$, наибольшее значение $D=350 \mathrm{kOe}$, а наименьшее $D=54 \mathrm{kOe}$. Наибольшее значение $A_{1}=300 \mathrm{kOe}$, наименьшее значение $A_{1}=50 \mathrm{kOe}$. Наибольшее значение $E=1500 \mathrm{kOe}$, а наименьшее значение $E=480 \mathrm{kOe}$.

Это обстоятельство затрудняет численное решение уравнения (9). Поэтому, используя формулу [8] для критического поля

$$
H_{c}=\sqrt{\frac{E}{G}\left(A_{1} E-D^{2}\right)+A_{1} E}-D
$$

и определяя из этой формулы $A_{1} E$, а затем подставляя это значение в (9), получаем [22]

$$
\begin{aligned}
\cos \theta[ & \left.-\frac{H^{2}}{H_{c}^{2}}-2 \frac{H D}{H_{c}^{2}} \cos \theta+2 \frac{D^{2}}{H_{c}^{2}} \sin ^{2} \theta+1+2 \frac{D}{H_{c}}\right] \\
& +\frac{H D}{H_{c}^{2}} \sin ^{2} \theta=0
\end{aligned}
$$

Поскольку значение $H_{c}$ определяется в эксперименте без проблем, задавая наименьшее $D=50 \mathrm{kOe}$ и наибольшее значение $D=350 \mathrm{kOe}$, мы нашли численные решения уравнения (11) [22]. С использованием этих решений в окрестности поля $H_{c}$ определены зависимости намагниченности от поля в угловой фазе [22]. Оказалось, что найденные зависимости намагниченности лучше соответствуют экспериментальным данным при $D=50 \mathrm{kOe}$. Поэтому, подставляя определенное значение для $A_{1} E$ в уравнение $(8)$, получаем

$$
\begin{aligned}
\frac{1}{\sqrt{2}} & \frac{H_{x}}{H_{c}}\left[\left(1+\frac{E}{G}\right) \frac{D}{H_{c}} \cos \theta+\frac{H_{z}}{H_{c}} \cos 2 \theta\right] \\
+ & \sin \theta\left\{\operatorname { c o s } \theta \left[-\frac{H_{z}^{2}}{H_{c}^{2}}-2 \frac{H_{z} D}{H_{c}} \cos \theta\right.\right. \\
& \left.\left.+2 \frac{D^{2}}{H_{c}^{2}} \sin ^{2} \theta+1+2 \frac{D}{H_{c}}\right]+\frac{H_{z} D}{H_{c}^{2}} \sin ^{2} \theta\right\}=0 .
\end{aligned}
$$

Поскольку обменные константы $E$ и $G$ различаются незначительно, можно положить $E=G$ и решать уравнение (12) при $D=50 \mathrm{kOe}$ и $H_{c}=210 \mathrm{kOe}$. Численные решения уравнения (4) выполнены при различных значениях магнитного поля в интервале от $H / H_{c}=0.99$ до 1.01 и значениях угла $\psi$ между осью $C_{4}$ и вектором $\mathbf{H}$

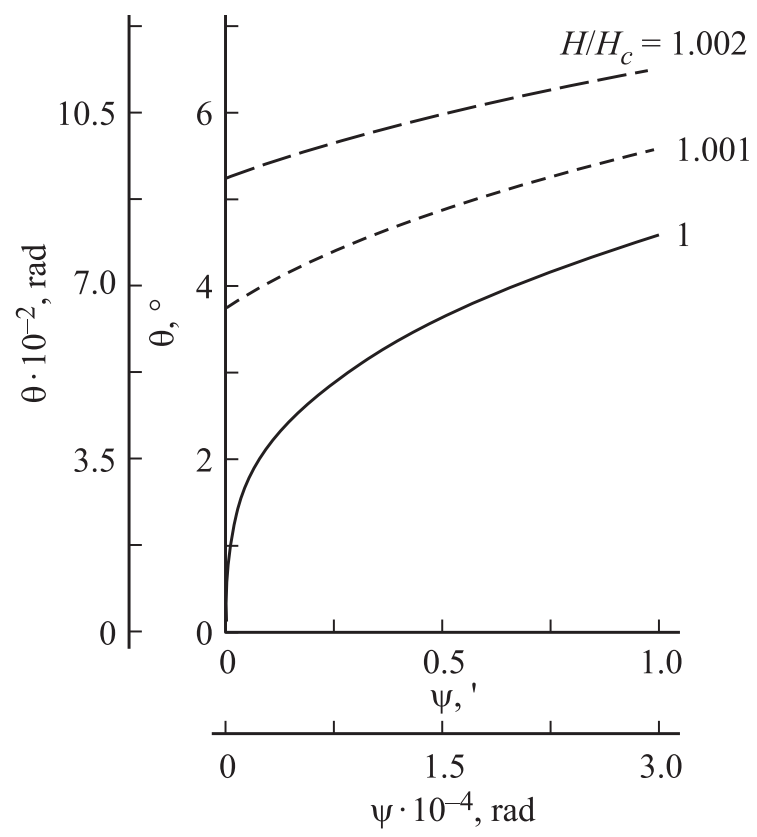

Рис. 1. Зависимость ориентации магнитной подсистемы кристалла $\mathrm{CoF}_{2}$ от ориентации магнитного поля $\mathbf{H}$ в полях $H / H_{c} \geq 1: \psi-$ угол между осью $C_{4}$ и направлением поля $\mathbf{H}$, $\theta-$ угол между осью $C_{4}$ и ориентацией вектора антиферромагнетизма $\mathbf{l}$.

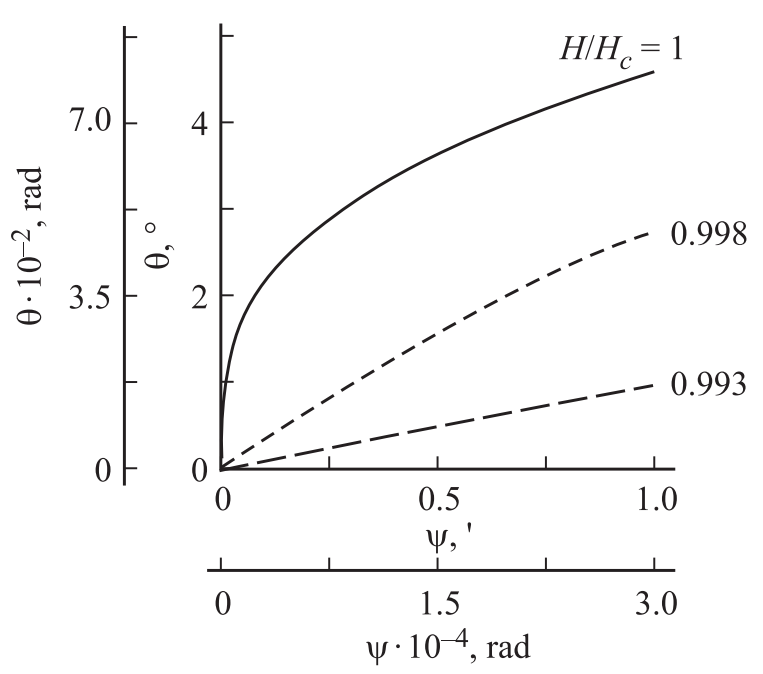

Рис. 2. Зависимость ориентации магнитной подсистемы кристалла $\mathrm{CoF}_{2}$ от ориентации магнитного поля $\mathbf{H}$ в полях $H / H_{c} \leq 1$. Обозначения те же, что на рис. 1 .

в интервале от $\psi=0$ до $1^{\circ}$. Поскольку использовалось условие $\varphi_{0} \ll 1$, в уравнении (12) было положено $H_{x}=H \sin \psi=H \psi$ и $H_{z}=H \cos \psi=H$. Наиболее важные результаты приведены на рис. 1 и 2.

Из приведенных данных следует, что наибольшее и значительное изменение ориентации магнитной подсистемы фторида кобальта с изменением ориентации поля $\mathbf{H}$ происходит при $H=H_{c}$. В этом случае при отклонении поля от оси $C_{4}$ всего на $1^{\prime}$ угол $\theta$ изменяется 
на $4.58^{\circ}$. При дальнейшем увеличении угла $\psi$ или увеличении или уменьшении величины поля всего на тысячные доли $H_{c}$ высокая чувствительность исчезает. Отметим, что обнаруженная ранее в работах $[23,24]$ аномалия в уменьшении эффективной магнитной анизотропии была связана с фазовым переходом первого рода, причем аномалия была тем сильнее, чем меньше отношение поля анизотропии к обменному полю. В данном же случае аномалия в уменьшении эффективной магнитной анизотропии связана с фазовым переходом второго рода и проявляется при необычайно большой магнитной анизотропии. Это обстоятельство представляет интерес в связи с многочисленными исследованиями соединений, обладающих гигантской магнитострикцией и огромной магнитной анизотропией.

В следующем разделе приведем несколько замечаний по использованию теории фазовых переходов Ландау в продольном магнитном поле.

\section{4. Теория Ландау в поле $\mathrm{H} \| \mathrm{C}_{4}$}

Используя плотность энергии в формуле (4) при $\varphi=\pi / 4$ и производя разложение термодинамических функций в ряд при $\theta \ll 1$, получаем

$$
\mathscr{H}=2 M_{0}\left(-\frac{H^{2}}{2 E}+A \theta^{2}+B \theta^{4}\right),
$$

где

$$
\begin{gathered}
A=\frac{G}{2 E(E+G)}\left[-H^{2}-2 H D+\frac{E+G}{G}\left(A_{1} E-D^{2}\right)\right], \\
B=\frac{G}{6 E(E+G)}\left[H^{2}+5 H D+3 D^{2}-\frac{E+G}{G}\left(A_{1} E-D^{2}\right)\right],
\end{gathered}
$$

а угол $\theta$ играет роль параметра порядка.

Исключая $A_{1} E$ из формул (14), (15) путем использования выражения для критического поля $H_{c}$, для коэффициентов $A$ и $B$ находим соотношения

$$
\begin{aligned}
& A=\frac{G}{2 E(E+G)}\left[-\left(H^{2}-H_{c}^{2}\right)-2 D\left(H-H_{c}\right)\right], \\
& B=\frac{G}{6 E(E+G)}\left[H^{2}-H_{c}^{2}+D\left(5 H+2 H_{c}\right)+3 D^{2}\right] .
\end{aligned}
$$

Поскольку $E, G, D>0$, имеем $B>0$, а коэффициент $A<0$ в несимметричной фазе $\left(\theta \neq 0, H>H_{c}\right)$ и $A>0$ в симметричной фазе $\left(\theta=0, H<H_{c}\right)$. В точке перехода $A=0\left(H=H_{c}\right)$. Отметим, что формула (14a) отличается от обычно более простого выражения $A \sim\left(T-T_{c}\right)$ (или $\left.A \sim\left(H-H_{c}\right)\right)$.

\section{5. Заключение}

Экспериментальную проверку вывода о существовании аномалии в уменьшении эффективной магнитной анизотропии можно осуществить, используя диэлектрический резонанс [25].
При изучении поглощения внешнего экспериментального поля на низких частотах можно также обнаружить максимум поглощения в окрестности поля $H_{c}$. По-видимому, представляет интерес экспериментальное изучение особенностей поглощения звука в кристалле $\mathrm{CoF}_{2}$ в окрестности $H_{c}$. Еще в работах [26-28] в кристаллах $\mathrm{MnF}_{2}, \mathrm{CrO}_{3}, \alpha-\mathrm{Fe}_{2} \mathrm{O}_{3}$ были обнаружены аномалии поглощения (и изменения скорости) поперечного и продольного звука. Для согласования различных механизмов поглощения звука, а также понимания причин теоретически предсказанных особенностей взаимодействия спиновых и звуковых волн в области ориентационных фазовых переходов [29-32] целесообразно изучить в эксперименте возможные поглощения звука в $\mathrm{CoF}_{2}$ при Н $\|$ А. Дело в том, что в работах [26-28] обнаруженные скачки поглощения звука зависели от направления волнового вектора k. Для поперечного звука они зависели еще и от направления вектора поляризации $\mathbf{l}_{t}$, тогда как пики поглощения в случае Н $\|$ А были обнаружены только для продольного звука и не зависели от направления волнового вектора $\mathbf{k}$.

\section{Список литературы}

[1] T.R. Dugan, J.M. Goldberg, W.W. Brennessel, P.L. Holland. Organometallics 31, 4, 1349 (2012).

[2] Y.T. Teng, S.S. Pramana, J. Ding, T. Wu, R. Yazami. Electrochim. Acta 107, 301 (2013).

[3] M.J. Armstrong, A. Panneerselvam, C. O'Regan, M.A. Morrisab, J.D. Holmes. J. Mater. Chem. A 1, 10667 (2013).

[4] C.Y. Lee, Z. Su, K. Lee, H. Tsuchiya, P. Schmuki. Chem. Commun. 50, 7067 (2014).

[5] M.C. Leclerc, J.M. Bayne, G.M. Lee, S.I. Gorelsky, M. Vasiliu, I. Korobkov, D.J. Harrison, D.A. Dixon, R.T. Baker. J. Am. Chem. Soc. 137, 16064 (2015).

[6] J. Tan, L. Liu, S. Guo, H. Hu, Z. Yan, Q. Zhou, Z. Huang, H. Shu, X. Yang, X. Wang. Electrochim. Acta 168, 225 (2015).

[7] Н.Ф. Харченко, В.В. Еременко, Л.И. Белый. ЖЭТФ 82, 827 (1982).

[8] К.Г. Гуртовой, А.С. Лагутин, В.И. Ожогин. ЖЭТФ 83, 1941 (1982).

[9] Q. Chu, Z. Xing, J. Tian, X. Ren, A.M. Asiri, A.O. Al-Youbi, K.A. Alamry, X. Sun. J. Power Sources 236, 188 (2013).

[10] J.H. Yang, Z.L. Li, X.Z. Lu, M.H. Whangbo, S.H. Wei, X.G. Gong, H.J. Xiang. Phys. Rev. Lett. 109, 107203 (2012).

[11] А.С. Прохоров, Е.Г. Рудашевский. Письма в ЖЭТФ 10, 175 (1969).

[12] Г.К. Чепурных, О.Г. Медведовская, О.А. Никитина. ФТТ 41, 2044 (1999).

[13] Г.К. Чепурных, О.Г. Медведовская, О.А. Никитина. ФНТ 26, 108 (2000).

[14] Z.Y. Jia, H.F. Liu, F.J. Wang, W. Liu, C.Y. Ge. Measurement 44, 88 (2011).

[15] J. Tamura, Y. Kawamura, H. Mochiji, N. Sasaki, K. Mizutani, H. Okawa. Jpn. J. Appl. Phys. 50, 07HC04 (2011).

[16] H. Liu, Z. Jia, F. Wang, F. Zong. Mechatronics 22, 911 (2012).

[17] К.П. Белов, Р.3. Левитин, С.А. Никитин. ФММ 11, 948 (1961). 
[18] S. Legvold, J. Alstad, J. Rhyne. Phys. Rev. Lett. 10, 509 (1963).

[19] К.П. Белов. Сорос. образоват. журн. 3, 15 (1998).

[20] N. Koon, A. Schinder, F. Carter. Phys. Lett. A 37, 413 (1971).

[21] J. Liu, T. Zhang, J. Wang, C. Jiang. Mater. China 4, 002 (2012).

[22] O.G. Medvedovs'ka, T.O. Fedorenko, G.K. Chepurnykh. Proc. of the XI Int. Conf. „Electronics and applied physics“. Kyiv, Ukraine (2015). P. 31.

[23] Г.К. Чепурных. ФТТ 10, 1917 (1968).

[24] М.И. Каганов, Г.К. Чепурных. ФТТ 11, 911 (1969).

[25] Н.К. Даньшин, Н.М. Ковтун, М.А. Сдвижков. ФТТ 26, 3635 (1984).

[26] Y. Shapira, J. Zak. Phys. Rev. 170, 503 (1968).

[27] Y. Shapira. Phys. Rev. 187, 734 (1969).

[28] Y. Shapira. Phys. Rev. 184, 589 (1969).

[29] С.В. Пелетминский. ЖЭТФ 37, 452 (1959).

[30] И.Е. Дикштейн, В.В. Тарасенко, В.Г. Шавров. ЖЭТФ 67, 816 (1974).

[31] Г.К. Чепурных. ФТТ 17, 430 (1975).

[32] Г.К. Чепурных. ФТТ 17, 2712 (1975). 